\title{
Salivary gland choriostoma in the esophagus
}

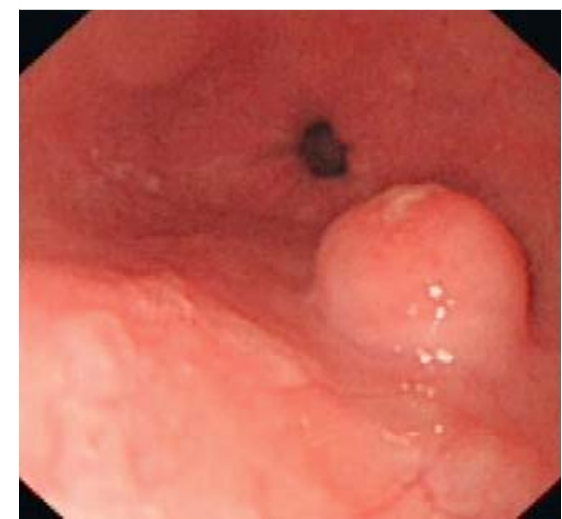

Fig. 1 Endoscopic image of an esophageal mucosal polypoid lesion in a 60-year-old man.

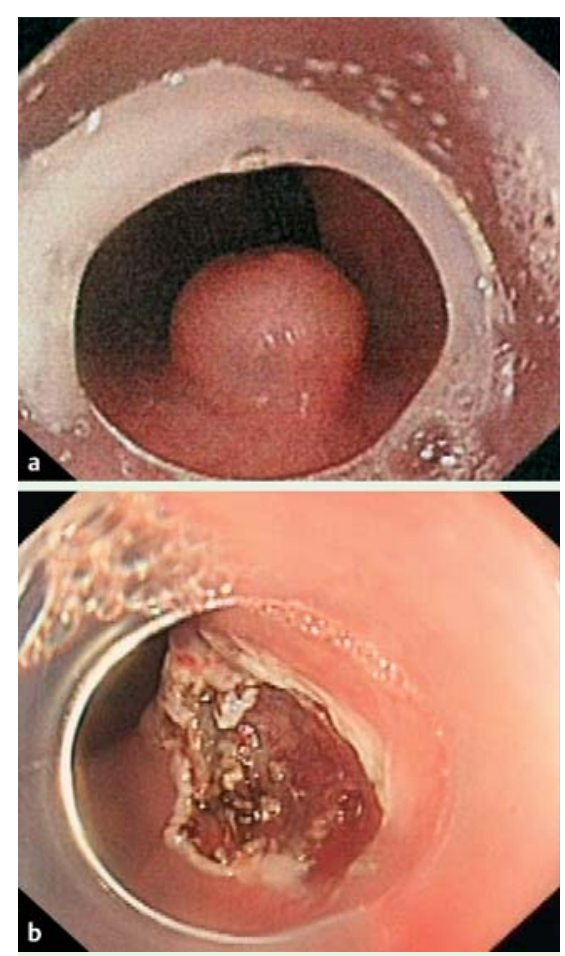

Fig. 3 Endoscopic images showing the endoscopic mucosal resection (EMR) with circumferential mucosal incision. a EMR was performed after the mucosa was sufficiently elevated by local injection. $\mathbf{b}$ The lesion was removed en bloc by EMR.

Salivary gland choriostoma is defined as a tumor-like growth of otherwise normal salivary gland found in an abnormal location. It has been reported in the head and neck regions but there are very few reports of its presence in the gastrointestinal tract $[1,2]$. To our knowledge, it has been reported in the jejunal [3], sigmoid

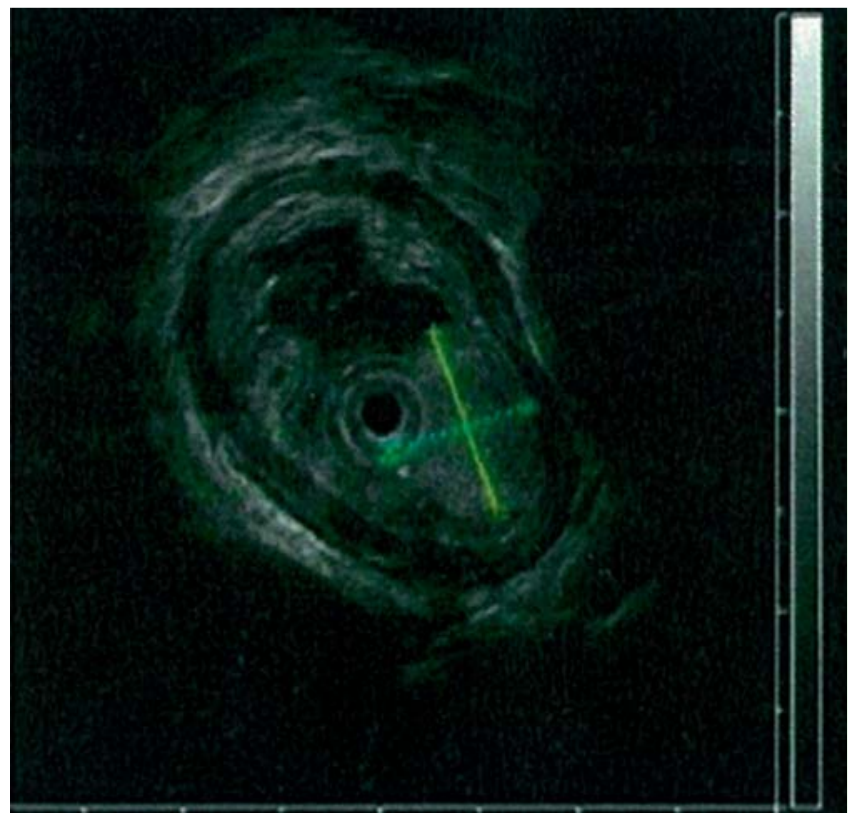

Fig. 2 Endoscopic ultrasound (EUS) image from the same patient showing an isohypoechoic, homogeneous lesion derived from the esophageal mucosal layer.

colon [4], rectal $[5,6]$, and perianal regions [7], but there is no report in the English literature of its presence in the esophagus. We report a unique case of esophageal mucosal salivary gland choriostoma in a 60-year-old man who underwent esophagogastroduodenoscopy for belch, regurgitation, and abdominal pain, and was treated by endoscopic resection.

Esophagogastroduodenoscopy revealed a $1.2 \times 1.0 \mathrm{~cm}$ mucosal protuberant lesion situated $38 \mathrm{~cm}$ from the incisors ( $\bullet$ Fig. 1). An ultrasound scan of the esophagus revealed that the mass, derived from the esophageal mucosal layer, was approximately $9.3 \times 7.5 \mathrm{~mm}$ ( Fig. 2 ). Initially, the mass was thought to be a polypus of esophageal mucosa. The patient underwent endoscopic mucosal resection (EMR) of the protuberant mass ( $\bullet$ Fig.3a,b).

At histological examination, the esophageal lesion was considered to be a salivary gland tumor, partly basal cell adenoma, partly with the structure of adenoid cystic carcinoma, and the margin was negative ( Fig.4a,b). With immunohistochemical staining, the glands were positive for CD117, P63, PDGFR, P53, Ki-67, CEA, P-CK, Vimentin, PAS, S-100, Calponin, and CK5/6.

In order to confirm whether the tumor was metastatic or not, the patient underwent bilateral parotid gland ultrasonography, but no obvious abnormalities were found. The patient was eventually diagnosed with primary esophageal salivary gland choriostoma.

In conclusion, we report an extremely rare case of salivary gland choriostoma in the esophagus. The literature describes some cases of heterotopic salivary gland tissue in the digestive tract, but the lesions were all in the lower digestive tract. Our case is different from previous reports as the lesion was located in the esophagus.

Endoscopy_UCTN_Code_CCL_1AB_2AC_3AH

\section{Competing interests: None}

\section{Changqing Wang, Ling Chen, Weiwei Guo, Xiaojuan Zhu, Zheng Liu}

Institute of Digestive Endoscopy and Medical Center for Digestive Diseases, Second Affiliated Hospital of Nanjing Medical University, Nanjing, China

\section{References}

1 Feller KU, Mavros A, Gaertner HJ. Ectopic submandibular thyroid tissue with a coexisting active and normally located thyroid gland: case report and review of literature. Oral Surg Oral Med Oral Pathol Oral Radiol Endod 2000; 90: 618-623

2 Mysorekar VV, Dandekar CP, Sreevathsa MR. Ectopic thyroid tissue in the parotid salivary gland. Singapore Med J 2004; 45: 437-438 


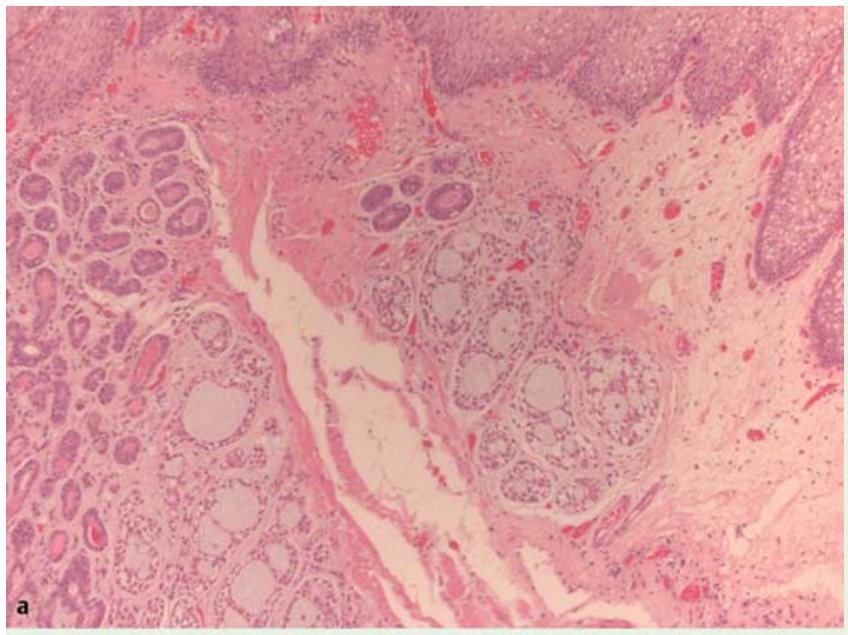

Fig. 4 Histological images of the esophageal lesion. a Hematoxylin and eosin staining of the primary esophageal salivary gland choriostoma (original magnification $\times 100)$. b Kit (CD117+) immunohistochemical staining was strongly positive (original magnification $\times 200)$.

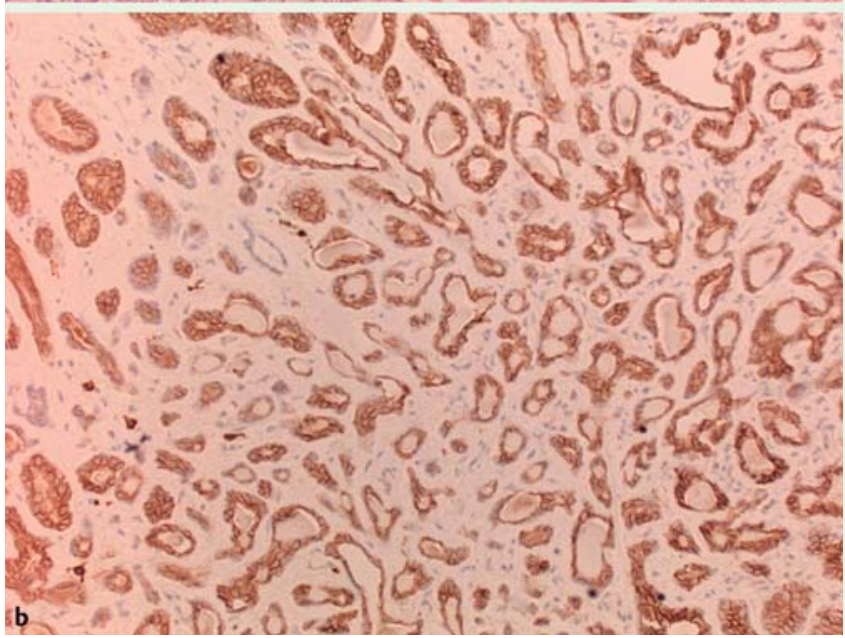

3 Olajide TA, Agodirin SO, Ojewola RW et al. Jejunal choristoma: a very rare cause of abdominal pain in children. Case Rep Surg 2014; 2014: 863647

4 Maffini F, Vingiani A, Lepanto D et al. Salivary gland choristoma in large bowel. Endoscopy 2012; 44 (Suppl. 02): E13-E14

5 Weitzner S. Ectopic salivary gland tissue in submucosa of rectum. Dis Colon Rectum 1983; 26: 814-817

6 Downs-Kelly E, Hoschar AP, Prayson RA. Salivary gland heterotopia in the rectum. Ann Diagn Pathol 2003; 7: 124-126

7 Evans CS, Goldman RL. Seromucinous (salivary) ectopia of the perianal region. Arch Dermatol 1987; 123: 1277

\section{Bibliography}

DOI http://dx.doi.org/

10.1055/s-0034-1390842

Endoscopy 2014; 46: E658-E659

(c) Georg Thieme Verlag KG

Stuttgart · New York

ISSN 0013-726X

\section{Corresponding author \\ Zheng Liu}

Institute of Digestive Endoscopy and Medical Center for Digestive Diseases Second Affiliated Hospital of Nanjing Medical University

121 jiang Jia Yuan

Nanjing 210011

China

Fax: +86-25-58509931

liuzheng117@yeah.net 